\title{
Robot-Assisted Surgical Navigation in Urology
}

\author{
Toshiyuki CHINA*1), ShujI ISOTANI*2), SHINICHI HISASUE*1), SHIGEO HORIE*1) \\ *1) Department of Urology, Juntendo University Faculty of Medicine, Tokyo, Japan, *2) Department of Urology, Teikyo \\ University School of Medicine, Tokyo, Japan
}

\begin{abstract}
As a current topic of interest, we report the utility of robot-assisted surgical navigation, using da Vinci, which is currently in operation in our department. In Japan, the Pharmaceutical Affairs Law regarding robot-assisted surgery was approved in 2009, which enabled us to perform robot-assisted prostatectomy for prostate cancer with coverage under the health insurance system from April 2012. As many as 180 units of robotic surgical systems are in operation in Japan this year, making Japan a robotic surgery Mecca, second only to the United States. Japan is by far the number one robotic-surgery-operating country in Asia. At the time of robot-assisted partial nephrectomy, in order to perform selective renal artery shut-off precisely, we conduct functional analysis of a medical image preoperatively, which provides us with a great deal of anatomical information during the operation. More specifically, a life-sized kidney model made by a 3D printer is very useful for this purpose. This model is based on the CT volume rendering image, and the 3D printer adopts a color-coded system in which tumor, kidney, and blood vessels are displayed with different colors. Surgical navigation and the 3D printer model in the case of partial nephrectomy enhanced our surgical performance and improved surgical safety.
\end{abstract}

Key words: surgical navigation, 3D printer model, robot-assisted partial nephrectomy, da Vinci, robotassisted prostatectomy

\section{Introduction}

First of all, I would like to briefly describe the history of surgery support robot da Vinci. Da Vinci was first engineered at Stanford Research Institute in the US in 1980s. In 1999, Intuitive Surgical, Inc. has announced the da Vinci system received the approval from the FDA, and in the following year, it was allowed to be used in general laparoscopic

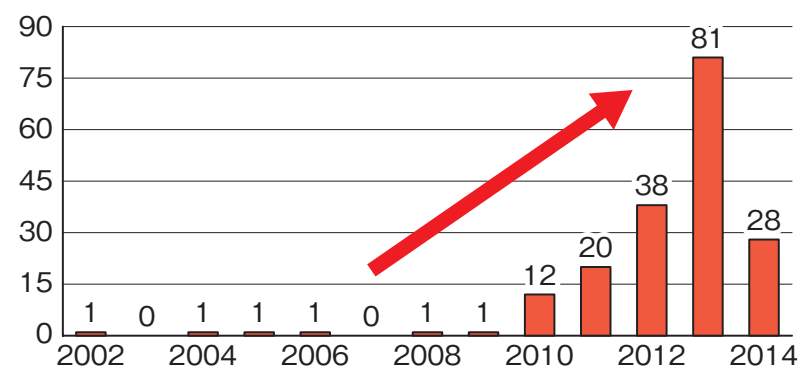

Figure-1 Robot-assisted surgery in Japan surgery. In Japan, Pharmaceutical Affairs Law was approved in 2009. This enabled us to perform robotassisted prostatectomy for prostate cancer within the range of health insurance by April 2012. Da Vinci system was employed in a countless number

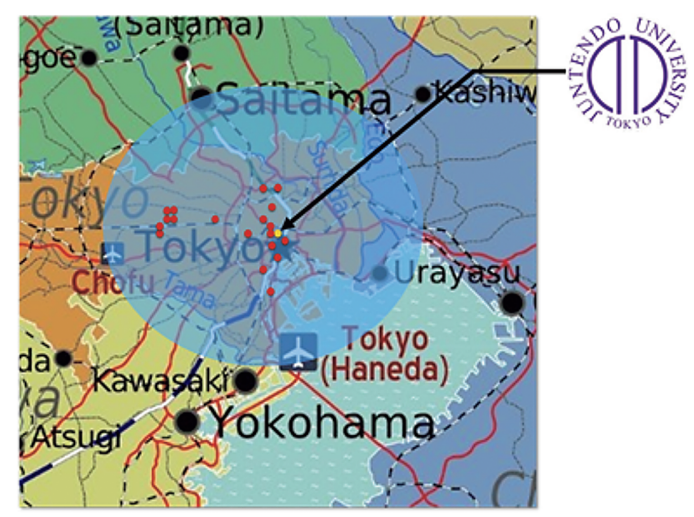

Figure-2 Robot-assisted surgery in Tokyo

Corresponding author: Toshiyuki China

Department of Urology, Juntendo University Faculty of Medicine

2-1-1 Hongo, Bunkyo-ku,Tokyo 113-8421, Japan

TEL: +81-3-3813-3111 E-mail: tchina@juntendo.ac.jp

334th Triannual Meeting of the Juntendo Medical Society: Future Perspectives of Robotic Surgery in Juntendo〔Held on Sep. 11, 2014〕

[Received Nov. 17, 2014] 
of urological operations, and the number of da Vinci machinery installed in medical facilities is now more than 3,000 units all over the world. In terms of the number, number one is undoubtedly the United States owing more than 2,000 units. Asian countries including Japan own more than 300 units. The number of surgical operations by da Vinci has increased year after year and exceeded the annual 500,000 at this point, mainly in area of gynecology, urology. These two account for the majority of proportion.

Da Vinci exerts its effect, presumably because it is used in a narrow pelvic cavity where is otherwise difficult to grasp the correct angle of the suture device and forceps. In Japan, there have been more than 180 units to date, giving us the second place in the world, second only to the United States. Surely, we are by far the number one robotic-surgeryoperating country in Asia (Figure-1). Especially, an area within a diameter of 15 kilometers around Juntendo is one of the most "da-Vinci populated" area in Tokyo, since more than 20 units are working (Figure-2).

\section{Operational status of the da Vinci system in Department of Urology, Juntendo Hospital}

In Juntendo hospital, two da Vinci systems have been working since July 2013. Within duration of only one year, I have experienced as many as 155 cases of prostatectomy. The number of patients referred to our hospital for surgery has increased dramatically, most of which are referred from our branch hospital (Figure-3). Incidentally, the number of referred patients from branch hospital for operation exceeded the number of operation-eligible outpatients from our institution.

Right after introduction of da Vinci, we have adopted the air seal system for safe and efficient operation. In detail, during pneumoperitoneum air seal system automatically removes and filters the fumes emitted from electric knife to maintain a clear view, and also by adjusting the gas flow, it curbs fluctuation in intra-peritoneum pressure caused by the suction. As you can imagine from its name, it has no valve to create pneumoperitoneum, which enables us to put device in and out of the operation field smoothly.

Then, is there anything surgeons can do in order to brush up da Vinci skills beforehand? The answer is yes, it can be achieved through da Vinci simulator mounted on the console, by which we can simulate an operation day and night using a real da Vinci system.

Next, we would like to refer to results of robotassisted prostatectomy for prostate cancer, with regard to surgical complication and pathological diagnosis.
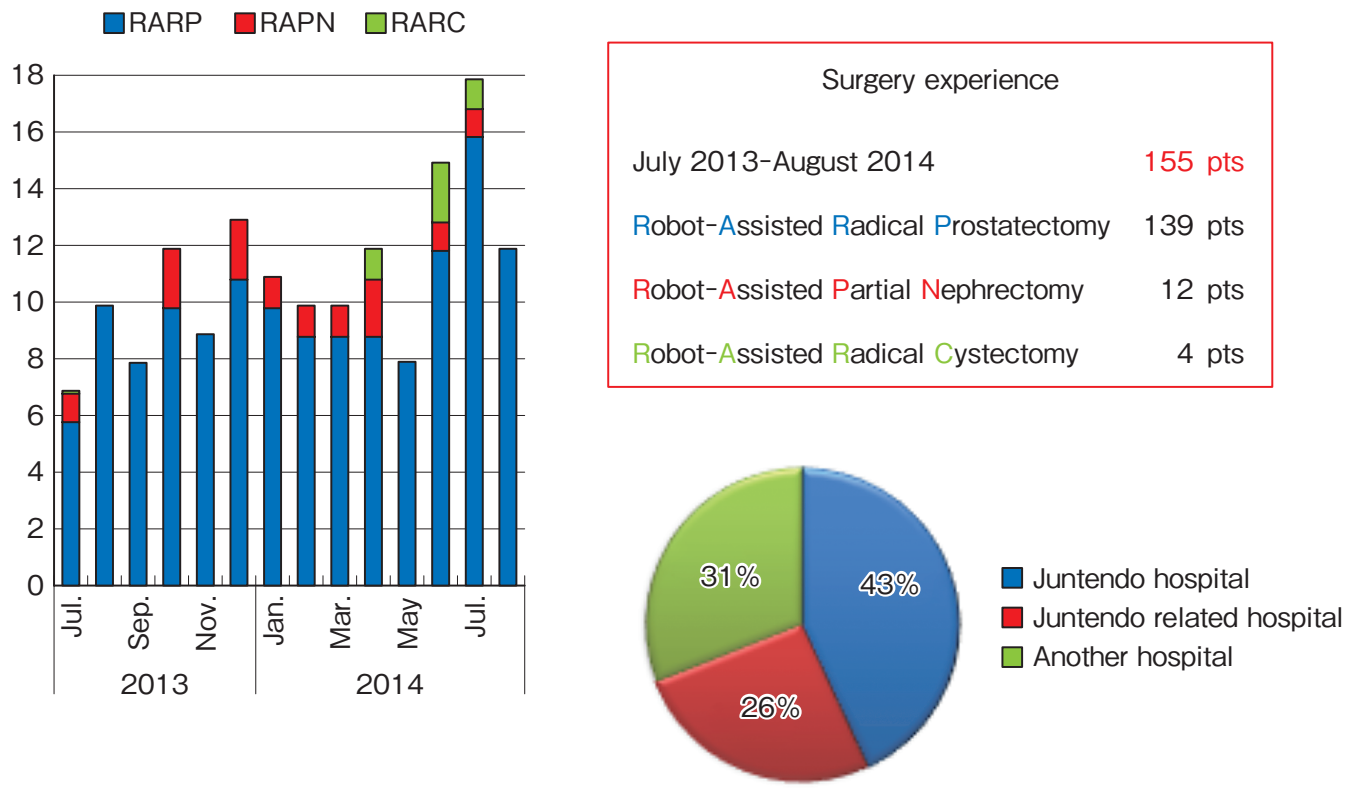

Figure-3 Robot-assisted surgery in Juntendo Hospital 


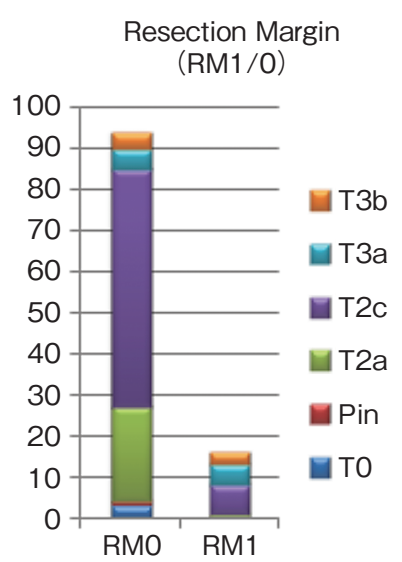

\begin{tabular}{|c|c|}
\hline \multicolumn{2}{|c|}{$\begin{array}{l}\text { Postoperative urinary } \\
\text { continence }\end{array}$} \\
\hline \multicolumn{2}{|c|}{$\begin{array}{c}\text { Pad } 1 \text { sheets or less / day } \\
\mathrm{n}=62 \text { (example observation period } 6 \text { months or more) }\end{array}$} \\
\hline$<$ POM 1 & 36 patients $(58 \%)$ \\
\hline$<$ POM 3 & 50 patients $(81 \%)$ \\
\hline$<$ POM 6 & 58 patients $(94 \%)$ \\
\hline
\end{tabular}

Figure-4 Robot-assisted prostatectomy

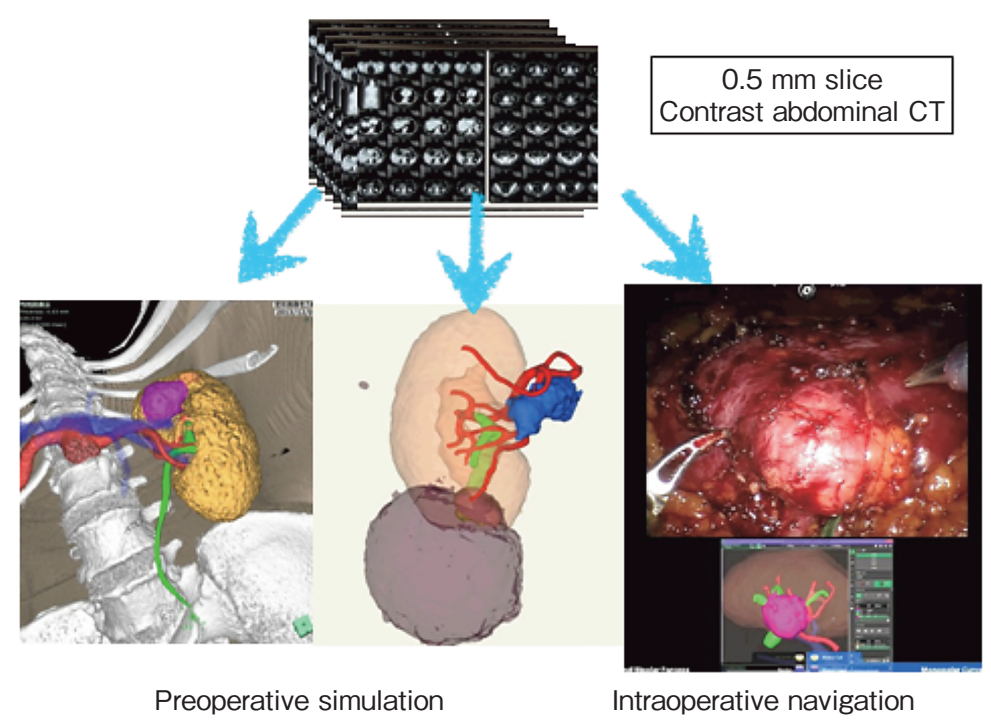

Figure-5 da Vinci surgical navigation

So-called trifecta for prostate cancer treatment are said to be anticancer, urinary continence, and erection. In terms of pathological diagnosis, positive margins rate is as low as $8 \%$ in $\mathrm{T} 1$ and $\mathrm{T} 2$ localized cancer. More than 80 percent of postoperative urinary incontinence showed a sign of improvement within 3 months after operation. All in all, we have achieved a very good result, which is compatible with the national and international reports (Figure-4).

\section{da Vinci surgical navigation and 3D printer model in robot-assisted partial nephrectomy}

At the time of robot-assisted partial nephrectomy, in order to perform precisely selective renal artery shut-off, we conduct functional analysis of a medical image preoperatively, which provides us with a great deal of anatomical information during operation. To do this, we carry out mainly the following four steps (Figure-5).

We will explain about each step in detail later in the chapter. Step 1: Determination of tumor resection range according to the position and depth of the tumor, Step 2: Identification and determination of clamp location of the tumor feeding renal artery, Step 3: The speculation about the possibility of renal pelvic section at the time of tumor resection, Step 4: Utilization of 3D image analysis system. For Step 4, we adopted synapse Vincent developed by Fuji film. This system is capable of rendering CT images to three-dimensional model. One of the 


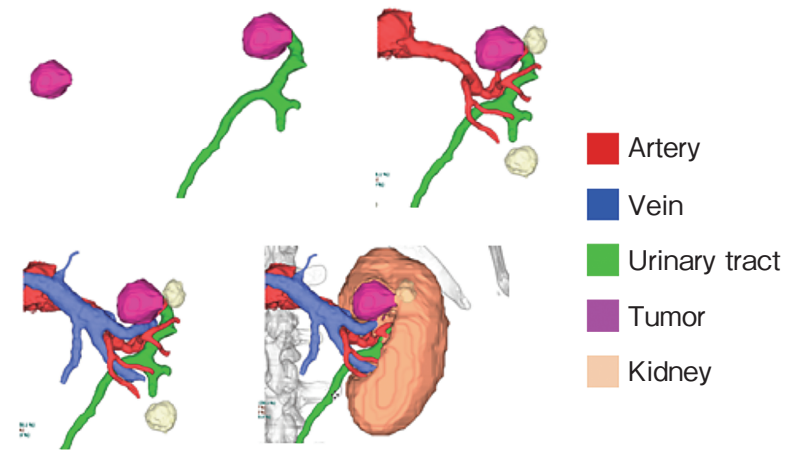

Figure-6 Rebuild the anatomical structure of the kidney as one of the $3 \mathrm{D}$ image

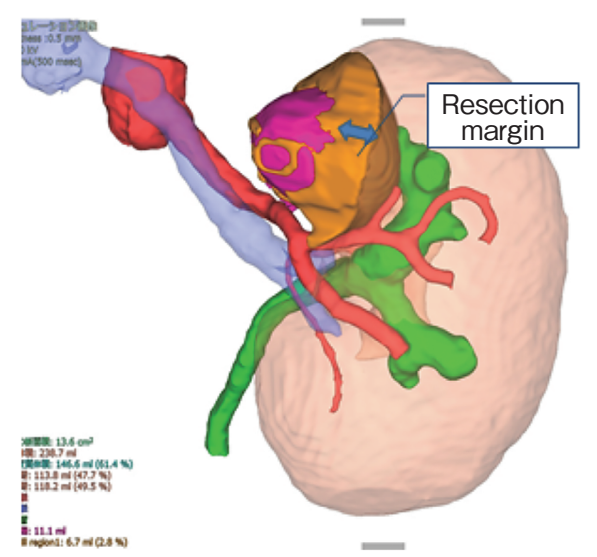

Figure-8 Step 1: Determination of tumor resection margin (Virtual Partial Nephrectomy)

distinctive features is that Vincent can incorporate the CT images from the early arterial phase until urine excretion phase to create a single three-dimensional model. Shown in Figure-6 is a virtual 3D model that was created by this procedure. In this model, different tissues are color-coded as follows: the artery red, veins blue, urinary tract green, the tumor purple, all of which are smartly installed in the semi-transparent brown kidney (Figure-6).

By segmenting each of the anatomical structure of the kidney this way, it enables us to see clear the positional relationship (Figure-7).

To determine tumor resection range in step 1 as mentioned above, we demarcate purple tumor and surrounding normal brown tissue leaving $5 \mathrm{~mm}$ of intact margin around tumor to avoid the exposure of tumor at resection surface (Figure-8).

Identification of the tumor feeding artery in step 2 and the determination of the renal artery clamping locations are instrumental to surmise blood flow control area of each arterial branch. For

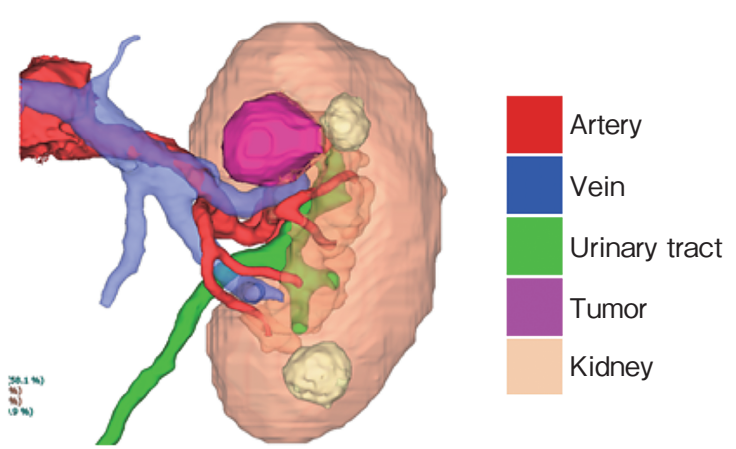

Figure -7 3D image that was segmented anatomical structure of the kidney
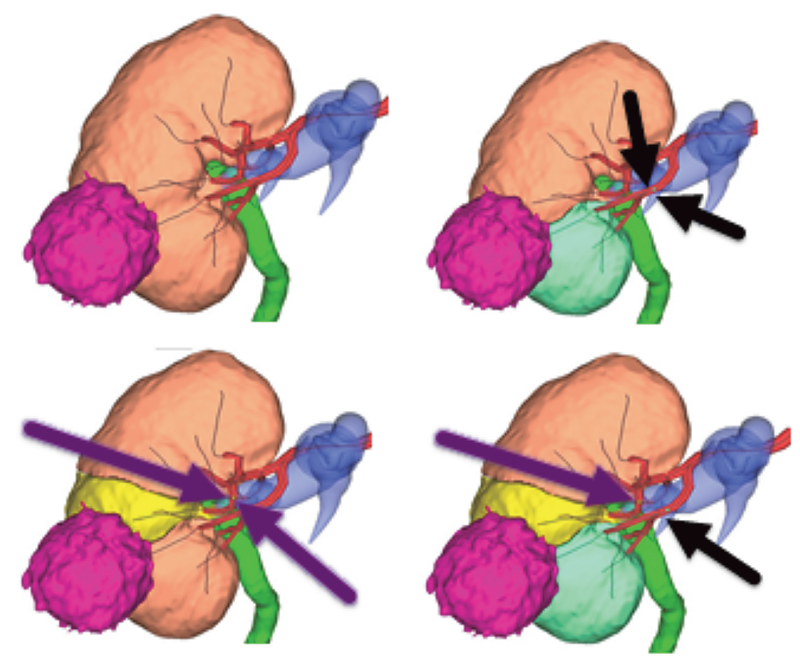

Figure-9 Step 2: Determination of the identification of tumor feeding artery and renal artery clamp location

example, as demonstrated in Figure-9, we identify two plausible nutrient arteries feeding a tumor as follows: black arrow indicates the one that governs the inferior pole of kidney, and purple arrow indicates the one that governs middle part of the kidney. This information allowed us to determine actual clamping locations of these two arteries.

We next move on to step 3, evaluation of possibility of pelvic section at the time of tumor resection. The left model in Figure-10 is a preoperative model with demarcation line of tumor resection created in step 1 , and the right model is putative postoperative model. Urinary tract pointed by a green arrow is in close proximity with renal pelvis. Therefore, we surmised that renal pelvic section might be necessary in this case. All of these information made us brace for prompt placement of intraoperative indwelling ureteral stent (Figure-10). 

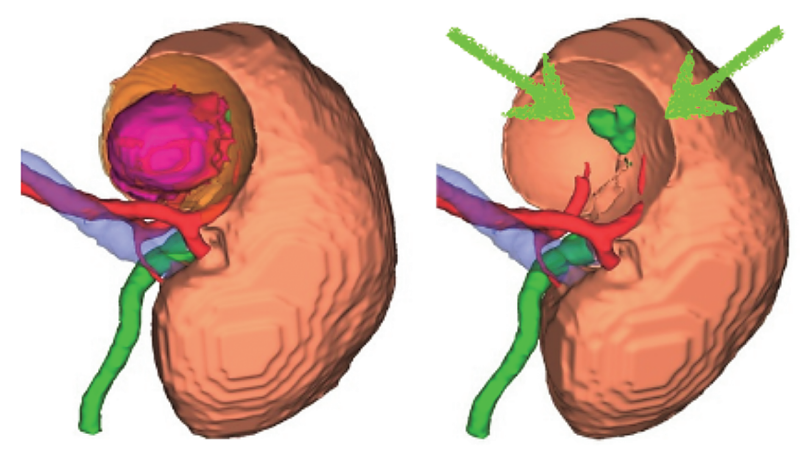

Figure-10 Step 3: Prediction of urinary tract open

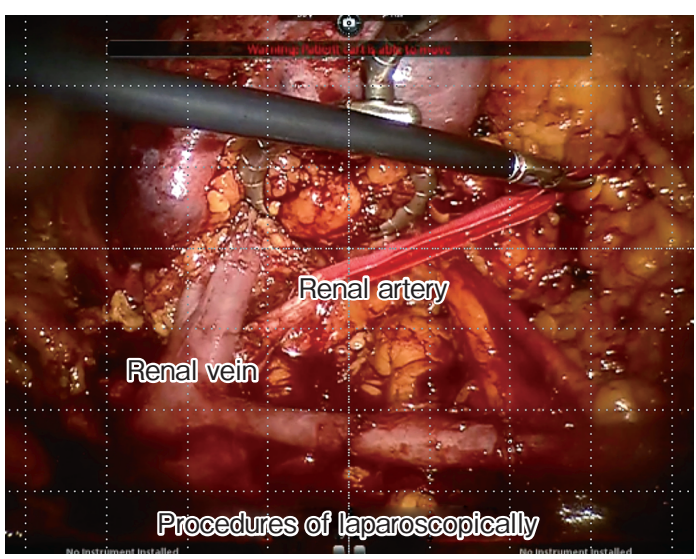

Figure-12 Procedures of laparoscopically

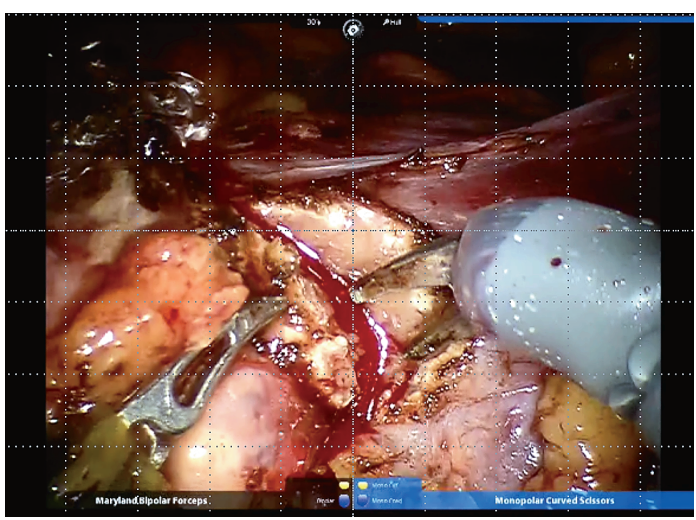

Figure-14 Partial nephrectomy

Now we move onto intraoperative navigation step 4 . The patient was 56-year-old man, with a tumor of $3 \mathrm{~cm}$ in diameter in the very inner rear surface of the left kidney. Although it is small in diameter, more than $50 \%$ of the tumor is buried to the renal parenchyma. Taken together, tumor is located in the very tricky place to perform partial nephrectomy in close contact with the urinary tract and renal vasculature (Figure-11).
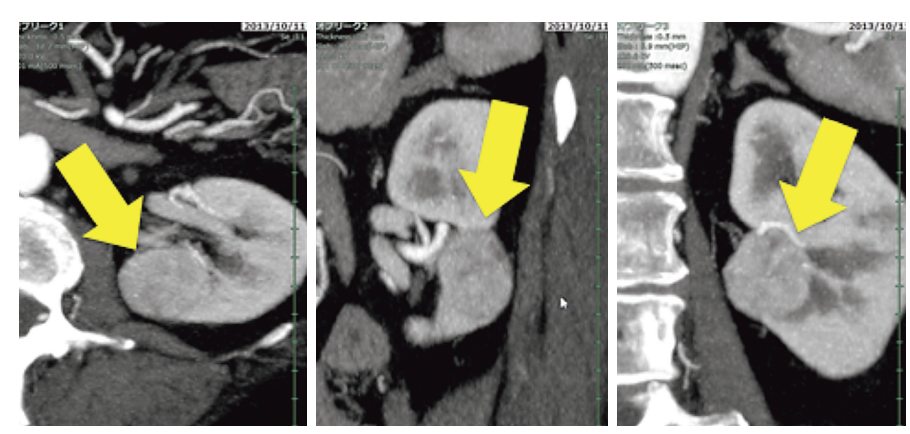

Figure-11 Step 4: Intraoperative navigation

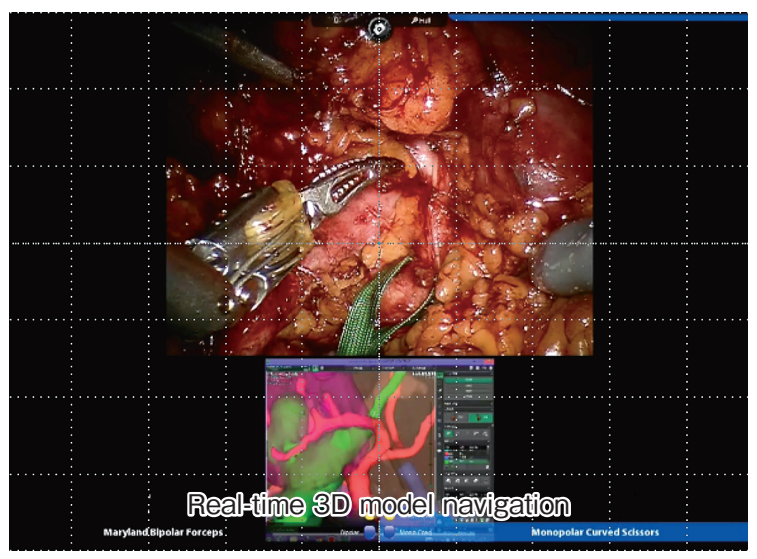

Figure-13 Real-time 3D model navigation

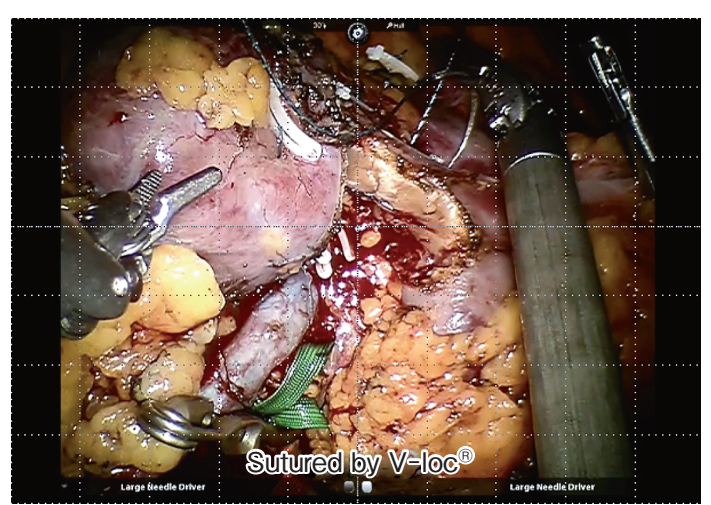

Figure-15 Suture of resection line

First, prior to performing the robotic surgery, we perform so-called peeling, exposure of the perirenal and renal vasculature to laparoscopically (Figure12). Then, the process proceeds to robotic surgery, where the guide operator provides the main operator with anatomical information using navigation screen of 3D model in real time.

Even after the tumor was identified, it is possible to bring the 3D model to the actual operative field 
(Figure-13). Moreover, we utilize intraoperative ultrasound to carefully determine the resection line. Here again, preoperative simulation helps us excise a tumor without hesitation, hence, it reduces the duration of the renal artery clamp, which is conducive to retention of renal function after all (Figure-14). Robotic surgery is capable of wide range of motion and dexterous movement using a needle that requires advanced technique in laparoscopy (Figure-15). In the near future, hopefully, the combination of the $3 \mathrm{D}$ model and robotic surgery give us perfect intraoperative real navigation.

\section{D printer model}

In the kidney of actual size created by 3D model

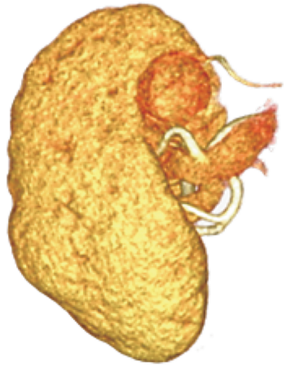

3D model image

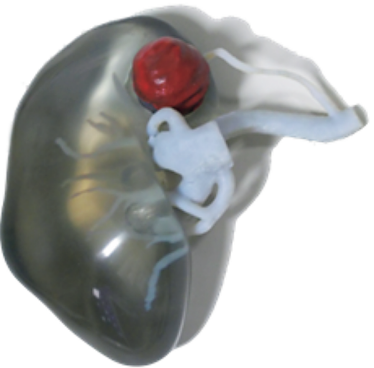

3D printer model
Figure-16 3D printer model

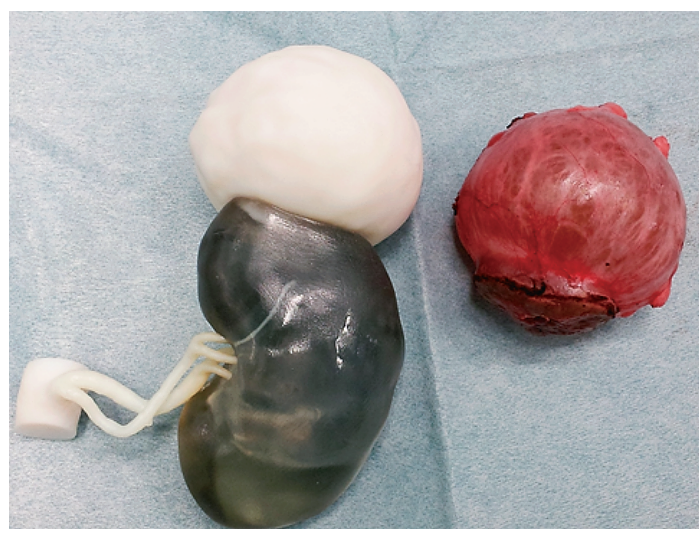

Figure-18 The 3D model replicate a real kidney printer shown in Figure-16, the tumor is color-coded in red, kidney is transparent, and the vascular system is segmented in white (Figure17). Figure-18 is a comparison of photograph between actual excised tumor and 3D printer model. It is surprising how the 3D model replicate a real kidney in both size and shape (Figure-18).

\section{Conclusion}

Surgical navigation and 3D printer model is very powerful tool when performing preoperative planning of partial nephrectomy. It guarantees safer operation and curtailed operation time.

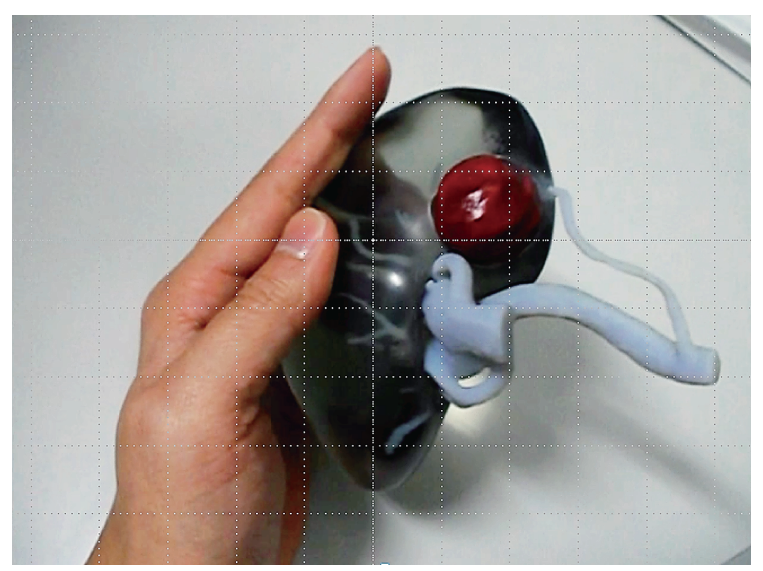

Figure-17 Segmented by color 